\title{
Identities associated with Milne-Thomson type polynomials and special numbers
}

\section{Yilmaz Simsek ${ }^{1}$ and Nenad Cakic ${ }^{2^{*}}$ (D)}

"Correspondence: cakic@etf.rs

2 Department of Mathematics, Faculty of Electrical Engineering, University of Belgrade, Belgrade, Serbia

Full list of author information is available at the end of the article

\section{Springer}

\begin{abstract}
The purpose of this paper is to give identities and relations including the Milne-Thomson polynomials, the Hermite polynomials, the Bernoulli numbers, the Euler numbers, the Stirling numbers, the central factorial numbers, and the Cauchy numbers. By using fermionic and bosonic $p$-adic integrals, we derive some new relations and formulas related to these numbers and polynomials, and also the combinatorial sums.

MSC: $05 \mathrm{~A} 15 ; 05 \mathrm{~A} 10 ; 11 \mathrm{~B} 68 ; 11 \mathrm{~B} 83$

Keywords: Generating function; Functional equation; Bernoulli numbers and polynomials; Euler numbers and polynomials; Stirling numbers; Array polynomials; Milne-Thomson polynomials; Hermite polynomials; Central factorial numbers; Cauchy numbers; Special functions; $p$-adic integral
\end{abstract}

\section{Introduction}

Recently, many authors have studied special numbers and polynomials with their generating functions. Because these special numbers and polynomials including the Bernoulli numbers and polynomials, the Euler numbers and polynomials, the Stirling numbers, the Milne-Thomson numbers and polynomials, the Hermite numbers and polynomials, Central factorial numbers, Cauchy numbers, and the others have many applications not only in mathematics, but also in other related areas. It is well-known that there are also many combinatorial interpretations of these special numbers especially, the Stirling numbers and the central factorial numbers in partition theory, in set theory, in probability theory and in other sciences. For combinatorial interpretations of these special numbers and polynomials with their generating functions see for details [1-31], and the references therein.

In this paper the following notation is used.

$\mathbb{N}=\{1,2,3, \ldots\}, \mathbb{N}_{0}=\{0,1,2,3, \ldots\}=\mathbb{N} \cup\{0\}$ and $\mathbb{Z}$ denotes the set of integers, $\mathbb{R}$ denotes the set of real numbers and $\mathbb{C}$ denotes the set of complex numbers. Assuming that $\ln (z)$ denotes the principal branch of the multi-valued function $\ln (z)$ with the imaginary part $\operatorname{Im}(\ln (z))$ constrained by $-\pi<\operatorname{Im}(\ln (z)) \leq \pi$. Furthermore, $0^{n}=1$ if $n=0$, and, $0^{n}=0$ if 
$n \in \mathbb{N}$.

$$
\left(\begin{array}{l}
x \\
v
\end{array}\right)=\frac{x(x-1) \cdots(x-v+1)}{v !}=\frac{(x)_{v}}{v !}
$$

(cf. [1-31], and the references therein).

In order to prove identities, relations, formulas, and combinatorial sums related to the special numbers and polynomials of this paper, we need the following generating functions for these special numbers and polynomials including some basic properties of them.

The Bernoulli polynomials are defined by

$$
F_{B}(t, x)=\frac{t e^{x t}}{e^{t}-1}=\sum_{n=0}^{\infty} B_{n}(x) \frac{t^{n}}{n !}
$$

Substituting $x=0$ into (1), we have the Bernoulli numbers $B_{n}$ :

$$
B_{n}=B_{n}(0)
$$

The Euler polynomials are defined by

$$
F_{E}(t, x)=\frac{2 e^{x t}}{e^{t}+1}=\sum_{n=0}^{\infty} E_{n}(x) \frac{t^{n}}{n !}
$$

Substituting $x=0$ into (2), we have the Euler numbers $E_{n}$ :

$$
E_{n}=E_{n}(0)
$$

(cf. [1-31], and the references therein).

The array polynomials are defined by

$$
F_{A}(t, x, v)=\frac{\left(e^{t}-1\right)^{v} e^{x t}}{v !}=\sum_{n=0}^{\infty} S_{v}^{n}(x) \frac{t^{n}}{n !}
$$

where $v \in \mathbb{N}_{0}$. By (3), we have

$$
S_{v}^{n}(x)=\frac{1}{v !} \sum_{j=0}^{v}(-1)^{v-j}\left(\begin{array}{l}
v \\
j
\end{array}\right)(x+j)^{n}
$$

(cf. $[1,3,24,26])$. Substituting $x=0$ into (3), we have the Stirling numbers of the second kind $S_{2}(n, v)$ :

$$
S_{2}(n, v):=S_{v}^{n}(0)
$$

which defined by means of the following generating function:

$$
F_{S}(t, v)=\frac{\left(e^{t}-1\right)^{v}}{v !}=\sum_{n=0}^{\infty} S_{2}(n, v) \frac{t^{n}}{n !}
$$

where $v \in \mathbb{N}_{0}(c f .[3,5,7,24,30,31]$ and the references therein). 
The Stirling numbers of the first kind are defined by the following generating function:

$$
F_{S 1}(t, v)=\frac{(\log (1+t))^{v}}{v !}=\sum_{n=0}^{\infty} S_{1}(n, v) \frac{t^{n}}{n !}
$$

where $v \in \mathbb{N}_{0}$ (cf. [1-31], and the references therein).

The central factorial numbers $T(n, k)$ of the second kind are defined by means of the following generating function:

$$
F_{T}(t, k)=\frac{1}{(2 k) !}\left(e^{t}+e^{-t}-2\right)^{k}=\sum_{n=k}^{\infty} T(n, k) \frac{t^{2 n}}{(2 n) !} .
$$

By using (6), for $n, k \in \mathbb{N}, T(0, k)=T(n, 0)=0, T(n, 1)=1$ and also

$$
T(n, k)=\frac{1}{(2 k) !} \sum_{j=0}^{2 k}(-1)^{j}\left(\begin{array}{c}
2 k \\
j
\end{array}\right)(j-k)^{2 n}
$$

(cf. $[2,4,5,26,32]$, and the references therein).

Two-variable Hermite polynomials are defined by

$$
F(t, x, y: v)=e^{x t+y t^{\nu}}=\sum_{n=0}^{\infty} H_{n}^{(v)}(x, y) \frac{t^{n}}{n !}
$$

(cf. $[7,20,22])$.

Let $v$ be integer $v \geq 2$. The polynomials $H_{n}^{(v)}(x, y)$ are given by the following explicit formula:

$$
H_{n}^{(v)}(x, y)=n ! \sum_{r=0}^{\left[\frac{n}{v}\right]} \frac{x^{n-v r} y^{r}}{r !(n-v r) !}
$$

(cf. [7, 20, 22]). Substituting $y=-1$ and $v=2$ into (8), we have classical Hermite polynomials

$$
H_{n}(x)=H_{n}^{(2)}(x,-1)
$$

(cf. $[7,20,22])$.

The Hermite numbers are defined by

$$
F_{H}(t)=e^{-t^{2}}=\sum_{n=0}^{\infty} H_{n} \frac{t^{n}}{n !}
$$

(cf. $[7,10,20,22])$.

Three-variable polynomials $y_{6}(n ; x, y, z ; a, b, v)$ are defined by the first author [29] as follows:

$$
\mathcal{G}(t, x, y, z ; a, b, v)=(b+f(t, a))^{z} e^{x t+y h(t, v)}=\sum_{n=0}^{\infty} y_{6}(n ; x, y, z ; a, b, v) \frac{t^{n}}{n !}
$$


where $f(t, a)$ is a member of family of analytic functions or meromorphic functions, $a$ and $b$ are any real numbers, $v$ is positive integer. When $x=0, y=z=1$, Equation (10) reduces to the following numbers:

$$
y_{6}(n ; 0,1,1 ; a, b, v)=y_{6}(n ; a, b, v)
$$

which defined by means of the following generating function:

$$
\mathcal{J}(t ; a, b, v)=(b+f(t, a)) e^{h(t, v)}=\sum_{n=0}^{\infty} y_{6}(n ; a, b, v) \frac{t^{n}}{n !}
$$

(cf. [29]). When $b=0$, in the numbers $y_{6}(n ; a, b, v)$ reduce to the Milne-Thomson numbers of order $a, \phi_{n}^{(a)}$ :

$$
y_{6}(n ; a, 0, v)=\phi_{n}^{(a)}
$$

(cf. [19, p. 514, Eq-(2)], [9, 29]). Substituting $z=1$ and $b=0$ into (10), we have

$$
y_{6}(n ; x, y, 1 ; a, 0, v)=\Psi_{n}^{(a)}(x, y, v)
$$

which is related to a relation between the $y_{6}(n ; x, y, z ; a, b, v)$ and the generalized MilneThomson's polynomials [6]. Setting $z=1, b=0, y=1$ and $h(t, 0)=g(t)$ into (10), we also have a relation between the $y_{6}(n ; x, y, z ; a, b, v)$ and the Milne-Thomson polynomials $\Phi_{n}^{(a)}(x)$ :

$$
y_{6}(n ; x, 1,1 ; a, 0,0)=\Phi_{n}^{(a)}(x)
$$

(cf. [19, 29]). When $z=1, b=0, y=1, f(t, a)=1$ and $h(t, v)=-\frac{v t^{2}}{2}$, Equation (10) reduces to the Hermite polynomials $H_{n}^{(v)}(x)$ :

$$
y_{6}(n ; x, 1,1 ; a, 0, v)=H_{n}^{(v)}(x)
$$

(cf. $[10,20,22,29])$ and also the Hermite numbers

$$
y_{6}(2 n ; 0,1,1 ; a, 0,2)=H_{2 n}=\frac{(-1)^{n}(2 n) !}{n !}
$$

for $n \geq 0(c f .[10,20,22])$.

\section{$1.1 p$-adic integral}

Here, we survey some properties of the $p$-adic integral. Thus, we give some notations and definitions. $\mathbb{Z}_{p}$ denotes the set of $p$-adic integers, $\mathbb{Q}_{p}$ denotes the set of $p$-adic rational numbers, $\mathbb{K}$ denotes a field with a complete valuation and $\mathbb{C}_{p}$ is completion of the algebraic closure of $\mathbb{Q}_{p} . C^{1}\left(\mathbb{Z}_{p} \rightarrow \mathbb{K}\right)$ denotes the set of continuous derivative functions. Let $f(x) \in$ $C^{1}\left(\mathbb{Z}_{p} \rightarrow \mathbb{K}\right)$. Kim [14] defined the $p$-adic $q$-integral as follows:

$$
I_{q}(f(x))=\int_{\mathbb{Z}_{p}} f(x) d \mu_{q}(x)=\lim _{N \rightarrow \infty} \frac{1}{\left[p^{N}\right]_{q}} \sum_{x=0}^{p^{N}-1} f(x) q^{x},
$$


where $q \in \mathbb{C}_{p}$ with $|1-q|_{p}<1, f \in C^{1}\left(\mathbb{Z}_{p} \rightarrow \mathbb{K}\right)$,

$$
[x]=[x: q]= \begin{cases}\frac{1-q^{x}}{1-q}, & q \neq 1, \\ x, & q=1\end{cases}
$$

and

$$
\mu_{q}(x)=\mu_{q}\left(x+p^{N} \mathbb{Z}_{p}\right)=\frac{q^{x}}{\left[p^{N}\right]}
$$

is a $q$-distribution on $\mathbb{Z}_{p}(c f$. [14]).

Remark 1 If $q \rightarrow 1$, then (11) reduces to the Volkenborn integral (or so-called the bosonic integral):

$$
\lim _{q \rightarrow 1} I_{q}(f(x))=I_{1}(f(x))=\int_{\mathbb{Z}_{p}} f(x) d \mu_{1}(x)=\lim _{N \rightarrow \infty} \frac{1}{p^{N}} \sum_{x=0}^{p^{N}-1} f(x),
$$

where

$$
\mu_{1}(x)=\mu_{1}\left(x+p^{N} \mathbb{Z}_{p}\right)=\frac{1}{p^{N}}
$$

denotes the Haar distribution on $\mathbb{Z}_{p}(c f .[23])$, and the references therein).

Remark 2 If $q \rightarrow-1$, then (11) reduces to the fermionic $p$-adic integral:

$$
\lim _{q \rightarrow-1} I_{q}(f(x))=I_{-1}(f(x))=\int_{\mathbb{Z}_{p}} f(x) d \mu_{-1}(x)=\lim _{N \rightarrow \infty} \sum_{x=0}^{p^{N}-1}(-1)^{x} f(x)
$$

and

$$
\mu_{-1}(x)=\mu_{-1}\left(x+p^{N} \mathbb{Z}_{p}\right)=\frac{(-1)^{x}}{p^{N}}
$$

$(c f \cdot[13])$.

The Bernoulli numbers are also given by the following bosonic $p$-adic integral:

$$
B_{n}=\int_{\mathbb{Z}_{p}} x^{n} d \mu_{1}(x)
$$

$(c f .[14,23])$.

On the other hand, the Euler numbers are also given by the following fermionic $p$-adic integral:

$$
E_{n}=\int_{\mathbb{Z}_{p}} x^{n} d \mu_{-1}(x)
$$

(cf. [13]). 
The Daehee numbers $D_{n}$ are introduced by the following bosonic $p$-adic integral:

$$
D_{n}=\int_{\mathbb{Z}_{p}}(x)_{n} d \mu_{1}(x)=\sum_{k=0}^{n} S_{1}(n, k) B_{k}=\frac{(-1)^{n} n !}{n+1}
$$

(cf. [8, 11], [21, p. 45], and the references therein).

The Changhee numbers $C h_{n}$ are introduced by the following fermionic $p$-adic integral:

$$
C h_{n}=\int_{\mathbb{Z}_{p}}(x)_{n} d \mu_{-1}(x)=\sum_{k=0}^{n} S_{1}(n, k) E_{k}=(-1)^{n} 2^{-n} n !
$$

(cf. [12-14], and the references therein).

We now summarize the results of this paper as follows:

In Sect. 2, by using generating functions and their functional equations, we give some identities including the three-variable polynomials $y_{6}(n ; x, y, z ; a, b, v)$, the Hermite polynomials, the array polynomials and the Stirling numbers of the second kind.

In Sect. 3, by using $p$-adic integrals, we give some identities, combinatorial sums and relations related to the three-variable polynomials $y_{6}(n ; x, y, z ; a, b, v)$, the Bernoulli numbers, the Euler numbers, the Stirling numbers, the Cauchy numbers (or the Bernoulli numbers of the second kind) and other special numbers such as the Daehee numbers and the Changhee numbers.

In Sect. 4, by using generating functions associated with trigonometric functions and the central factorial numbers of the second kind, we derive identities related to the central factorial numbers of the second kind, the array polynomials, and combinatorial sum.

\section{Identities related to the Hermite polynomials, array polynomials and Stirling numbers of the second kind: generating functions and their functional equations approach}

In this section, by applying generating functions and their functional approach, we derive some identities including the three-variable polynomials $y_{6}(n ; x, y, z ; a, b, v)$, the Hermite polynomials, the array polynomials and the Stirling numbers of the second kind.

\section{Theorem 1}

$$
y_{6}(n ; x, y, 1 ; a, 0, v)=n ! \sum_{j=0}^{\left[\frac{n}{v}\right]} \frac{S_{a}^{n-v j}(x) y^{j}}{j !(n-v j) !}
$$

where $[x]$ denotes the greatest integer function.

Proof Substituting $b=0, h(t, v)=t^{v}$ and $f(t, a)=\frac{1}{a !}\left(e^{t}-1\right)^{a}$ with $a \in \mathbb{N}_{0}$ and $z=1$ into (10), we get the following functional equation:

$$
\mathcal{G}(t, x, y, 1 ; a, 0, v)=e^{y t^{v}} F_{A}(t, x, v) .
$$

Combining the above equation with (10), and (3), we obtain

$$
\sum_{n=0}^{\infty} y_{6}(n ; x, y, 1 ; a, 0, v) \frac{t^{n}}{n !}=\sum_{n=0}^{\infty} S_{a}^{n}(x) \frac{t^{n}}{n !} \sum_{n=0}^{\infty} \frac{y^{n} t^{v n}}{n !} .
$$


Now, combining the above equation with the following well-known series identity:

$$
\sum_{n=0}^{\infty} \sum_{k=0}^{\infty} A(n, k)=\sum_{n=0}^{\infty} \sum_{k=0}^{\left[\frac{n}{v}\right]} A(n, n-v k)
$$

(cf. [20, Lemma 11, Eq-(7)]), we get

$$
\sum_{n=0}^{\infty} y_{6}(n ; x, y, 1 ; a, 0, v) \frac{t^{n}}{n !}=\sum_{n=0}^{\infty}\left(n ! \sum_{j=0}^{\left[\frac{n}{v}\right]} \frac{S_{a}^{n-v j}(x) y^{j}}{j !(n-v j) !}\right) \frac{t^{n}}{n !}
$$

Equating the coefficients of $\frac{t^{n}}{n !}$ on both sides of the equation, we arrive at the desired result.

\section{Theorem 2}

$$
y_{6}(n ; x, y, 1 ; a, 0, v)=\sum_{j=0}^{n}\left(\begin{array}{l}
n \\
j
\end{array}\right) S_{2}(n-j, a) H_{j}^{(v)}(x, y) .
$$

Proof Proof of this theorem was also given in [33, Theorem 2]. We now briefly give another proof of this theorem. Substituting $b=0, h(t, v)=t^{v}$ and $f(t, a)=\frac{1}{a !}\left(e^{t}-1\right)^{a}$ with $a \in \mathbb{N}_{0}$ and $z=1$ into (10), we construct the following functional equation:

$$
\mathcal{G}(t, x, y, 1 ; a, 0, v)=F(t, x, y: v) F_{S}(t, a) .
$$

Combining the above equation with (10), (7), and (4), we get

$$
\sum_{n=0}^{\infty} y_{6}(n ; x, y, 1 ; a, 0, v) \frac{t^{n}}{n !}=\sum_{n=0}^{\infty} S_{2}(n, a) \frac{t^{n}}{n !} \sum_{n=0}^{\infty} H_{n}^{(v)}(x, y) \frac{t^{n}}{n !}
$$

Therefore

$$
\sum_{n=0}^{\infty} y_{6}(n ; x, y, 1 ; a, 0, v) \frac{t^{n}}{n !}=\sum_{n=0}^{\infty}\left(\sum_{j=0}^{n}\left(\begin{array}{l}
n \\
j
\end{array}\right) S_{2}(n-j, a) H_{j}^{(v)}(x, y)\right) \frac{t^{n}}{n !}
$$

Equating the coefficients $\frac{t^{n}}{n !}$ on both sides of the equation, we arrive at the desired result.

Combining (18) and (19), we arrive at the following theorem.

\section{Theorem 3}

$$
\sum_{j=0}^{n}\left(\begin{array}{l}
n \\
j
\end{array}\right) S_{2}(n-j, a) H_{j}^{(v)}(x, y)=n ! \sum_{j=0}^{\left[\frac{n}{v}\right]} \frac{S_{a}^{n-v j}(x) y^{j}}{j !(n-v j) !} .
$$




\section{Identities and relations related to Stirling numbers and other special numbers: $p$-adic integral approach}

In this section, by applying $p$-adic integrals approach, we derive some identities, combinatorial sums and relations related to the three-variable polynomials $y_{6}(n ; x, y, z ; a, b, v)$, the Bernoulli numbers, the Euler numbers, the Stirling numbers, the Cauchy numbers (or the Bernoulli numbers of the second kind) and other special numbers such as the Daehee numbers and the Changhee numbers.

Theorem 4 Let $m \in \mathbb{N}_{0}$ and $z \in \mathbb{R}$. We have

$$
y_{6}(m ; 0,0, z ; 1,1,1)=\sum_{n=0}^{m} S_{2}(m, n)(z)_{n}=z^{m} .
$$

Proof Substituting $a=b=v=1, x=y=0$ and $f(t, 1)=e^{t}-1$ into (10), we get

$$
\mathcal{G}(t, 0,0, z ; 1,1,1)=\left(1+\left(e^{t}-1\right)\right)^{z}=\sum_{n=0}^{\infty} y_{6}(n ; 0,0, z ; 1,1,1) \frac{t^{n}}{n !} .
$$

We assume that $\left|e^{t}-1\right|<1$. We obtain

$$
\sum_{m=0}^{\infty} y_{6}(m ; 0,0, z ; 1,1,1) \frac{t^{m}}{m !}=\sum_{n=0}^{\infty}(z)_{n} \frac{\left(e^{t}-1\right)^{n}}{n !}
$$

where

$$
(z)_{n}=z(z-1) \cdots(z-n+1) .
$$

Combining the above equation with (4), since $S_{2}(m, n)=0$ for $m<n$, we obtain

$$
\sum_{m=0}^{\infty} y_{6}(m ; 0,0, z ; 1,1,1) \frac{t^{m}}{m !}=\sum_{m=0}^{\infty}\left(\sum_{n=0}^{m} S_{2}(m, n)(z)_{n}\right) \frac{t^{m}}{m !} .
$$

Equating the coefficients of $\frac{t^{m}}{m !}$ on both sides of the equation, we arrive at the desired result.

It is time to give integral formulas for the three-variable polynomials $y_{6}(n ; x, y, z ; a, b, v)$. By applying the bosonic $p$-adic integral (or the Volkenborn integral) to (20), we obtain

$$
\int_{\mathbb{Z}_{p}} y_{6}(m ; 0,0, z ; 1,1,1) d \mu_{1}(z)=\sum_{n=0}^{m} S_{2}(m, n) \int_{\mathbb{Z}_{p}}(z)_{n} d \mu_{1}(z)
$$

By combining the above equation with (16), we obtain the following $p$-adic integral formulas, respectively:

$$
\begin{aligned}
& \int_{\mathbb{Z}_{p}} y_{6}(m ; 0,0, z ; 1,1,1) d \mu_{1}(z)=\sum_{n=0}^{m} S_{2}(m, n) D_{n}, \\
& \int_{\mathbb{Z}_{p}} y_{6}(m ; 0,0, z ; 1,1,1) d \mu_{1}(z)=\sum_{n=0}^{m}(-1)^{n} \frac{n ! S_{2}(m, n)}{n+1},
\end{aligned}
$$


and we also have

$$
\int_{\mathbb{Z}_{p}} y_{6}(m ; 0,0, z ; 1,1,1) d \mu_{1}(z)=\sum_{n=0}^{m} \sum_{j=0}^{n} S_{2}(m, n) S_{1}(n, j) B_{j} .
$$

Combining Eqs. (21) and (22), we get the following theorem.

Theorem 5 Let $m \in \mathbb{N}_{0}$. We have

$$
\sum_{n=0}^{m} \sum_{j=0}^{n} S_{2}(m, n) S_{1}(n, j) B_{j}=\sum_{n=0}^{m}(-1)^{n} \frac{n ! S_{2}(m, n)}{n+1} .
$$

We observe that, using the orthogonality relation of the Stirling numbers, Eq. (23) reduces to the following well-known relations for the Bernoulli numbers:

$$
B_{m}=\sum_{n=0}^{m}(-1)^{n} \frac{n ! S_{2}(m, n)}{n+1}
$$

(cf. $[3,7,11,17,18,25]$, and the references therein).

By applying the fermionic $p$-adic integral to (20), we obtain

$$
\int_{\mathbb{Z}_{p}} y_{6}(m ; 0,0, z ; 1,1,1) d \mu_{-1}(z)=\sum_{n=0}^{m} S_{2}(m, n) \int_{\mathbb{Z}_{p}}(z)_{n} d \mu_{-1}(z) .
$$

By combining the above equation with (17), we obtain

$$
\int_{\mathbb{Z}_{p}} y_{6}(m ; 0,0, z ; 1,1,1) d \mu_{-1}(z)=\sum_{n=0}^{m}(-1)^{n} \frac{n !}{2^{n}} S_{2}(m, n),
$$

and we also obtain

$$
\int_{\mathbb{Z}_{p}} y_{6}(m ; 0,0, z ; 1,1,1) d \mu_{-1}(z)=\sum_{n=0}^{m} \sum_{j=0}^{n} S_{2}(m, n) S_{1}(n, j) E_{j} .
$$

Combining the above equations, we get the following theorem.

Theorem 6 Let $m \in \mathbb{N}_{0}$. We have

$$
\sum_{n=0}^{m} \sum_{j=0}^{n} S_{2}(m, n) S_{1}(n, j) E_{j}=\sum_{n=0}^{m}(-1)^{n} \frac{n !}{2^{n}} S_{2}(m, n) .
$$

We also observe that, using the orthogonality relation of the Stirling numbers, Eq. (26) reduces to the following well-known relations for the Bernoulli numbers:

$$
E_{m}=\sum_{n=0}^{m}(-1)^{n} \frac{n !}{2^{n}} S_{2}(m, n)
$$

(cf. $[7,12,17,18,25]$, and the references therein). 
Integrating Eq. (20) with respect to $z$ from 0 to 1 , we obtain

$$
\int_{0}^{1} y_{6}(m ; 0,0, z ; 1,1,1) d z=\sum_{n=0}^{m} S_{2}(m, n) \int_{0}^{1}(z)_{n} d z
$$

Combining the above integral equation with the integral equation for the Cauchy numbers (the Bernoulli numbers of the second kind),

$$
b_{n}(0)=\int_{0}^{1}(z)_{n} d z
$$

(cf. [22, p. 114]), we get the following integral representation of the polynomials $y_{6}(m ; 0,0, z ; 1,1,1)$.

Corollary 1 Let $m \in \mathbb{N}_{0}$. We have

$$
\int_{0}^{1} y_{6}(m ; 0,0, z ; 1,1,1) d z=\sum_{n=0}^{m} S_{2}(m, n) b_{n}(0)
$$

\section{Identities including the central factorial numbers of the second kind and array polynomials}

In this section, by using special infinite series including trigonometric functions and the central factorial numbers of the second kind, we derive identities associated with the central factorial numbers of the second kind and the array polynomials.

In [2, Theorem 4.1.1 and Proposition 4.1.5], Butzer et al. gave the following alternative generating functions for the central factorial numbers of the second kind:

$$
\begin{aligned}
& \frac{1}{(2 m) !}\left(2 \sin \left(\frac{t}{2}\right)\right)^{2 m}=\sum_{n=0}^{\infty}(-1)^{n+m} T(2 n, 2 m) \frac{t^{2 n}}{(2 n) !}, \\
& \left(\cos \left(\frac{t}{2}\right)\right)^{2 m}=\sum_{n=0}^{\infty}(-1)^{n+m} T(2 n, 2 m) \frac{t^{2 n}}{(2 n) !} \sum_{j=0}^{m}\left(\begin{array}{c}
m \\
j
\end{array}\right) 4^{-j}(2 j) ! T(2 n, 2 j),
\end{aligned}
$$

$m \in \mathbb{N}_{0}$.

Since

$$
\sin (t)=\frac{e^{i t}-e^{-i t}}{2 i}
$$

where $i^{2}=-1$, after some elementary calculations, we obtain

$$
(\sin (t))^{2 m}=(2 m) ! \sum_{n=0}^{\infty}(-1)^{n-m}(i)^{n} 2^{n-2 m} S_{2 m}^{n}(-m) \frac{t^{n}}{n !} .
$$


Combining (27) and (28), and also using the Cauchy product formula for a series product, we also obtain

$$
\begin{aligned}
\left(2 \sin \left(\frac{t}{2}\right) \cos \left(\frac{t}{2}\right)\right)^{2 m}= & \sum_{n=0}^{\infty} \sum_{v=0}^{n}(-1)^{n+m}\left(\begin{array}{c}
2 n \\
2 v
\end{array}\right)(2 m) ! \\
& \times T(2 n-2 v, 2 m) \sum_{j=0}^{m}\left(\begin{array}{c}
m \\
j
\end{array}\right) 4^{-j}(2 j) ! T(2 v, 2 j) \frac{t^{2 n}}{(2 n) !} .
\end{aligned}
$$

Since

$$
2 \sin \left(\frac{t}{2}\right) \cos \left(\frac{t}{2}\right)=\sin (t)
$$

combining (29) and (30), after some calculations, we arrive at the following theorem including a relation between the central factorial numbers of the second kind and the array polynomials.

Theorem 7 For $m, n \in \mathbb{N}_{0}$ we have

$$
S_{2 m}^{2 n}(-m)=\sum_{v=0}^{n}\left(\begin{array}{c}
2 n \\
2 v
\end{array}\right) T(2 n-2 v, 2 m) \sum_{j=0}^{m}\left(\begin{array}{c}
m \\
j
\end{array}\right) 4^{n-m-j}(2 j) ! T(2 v, 2 j) .
$$

By combining (29) and (30), we also get following corollary.

Corollary 2 For $m, n \in \mathbb{N}_{0}$ we have

$$
S_{2 m}^{2 n+1}(-m)=0
$$

With the help of Eq. (31), we also obtain the following combinatorial sum.

Corollary 3 For $m, n \in \mathbb{N}_{0}$ we have

$$
\sum_{j=0}^{2 m} \sum_{k=0}^{2 n+1}(-1)^{-j-k}\left(\begin{array}{c}
2 m \\
j
\end{array}\right)\left(\begin{array}{c}
2 n+1 \\
k
\end{array}\right) j^{k} m^{2 n+1-k}=0
$$

\section{Conclusion}

This paper contains many kind of identities and relations related to the Milne-Thomson polynomials, the Hermite polynomials, the Bernoulli numbers, the Euler numbers, the Stirling numbers, the central factorial numbers, and the Cauchy numbers. By applying not only $p$-adic integral, but also the Riemann integral methods, many identities relations and formulas related to the aforementioned numbers and polynomials, and also the combinatorial sums are given. By using the orthogonality relation of the Stirling numbers, explicit formulas for the Bernoulli numbers and the Euler numbers are provided.

The results of this paper have potential applicability to physics, engineering and other related fields, especially branches of mathematics. 


\section{Acknowledgements}

We would like to thank the referees for their valuable comments. The first author was supported by the Scientific Research Project Administration of Akdeniz University.

\section{Competing interests}

The authors declare that they have no competing interests.

\section{Authors' contributions}

All the authors participated in every phase of the research conducted for this paper. All authors read and approved the final manuscript.

\section{Author details}

'Department of Mathematics, Faculty of Science, University of Akdeniz, Antalya, Turkey. ${ }^{2}$ Department of Mathematics, Faculty of Electrical Engineering, University of Belgrade, Belgrade, Serbia.

\section{Publisher's Note}

Springer Nature remains neutral with regard to jurisdictional claims in published maps and institutional affiliations.

\section{Received: 27 February 2018 Accepted: 3 April 2018 Published online: 13 April 2018}

\section{References}

1. Bayad, A., Simsek, Y., Srivastava, H.M.: Some array type polynomials associated with special numbers and polynomials. Appl. Math. Comput. 244, 149-157 (2014)

2. Butzer, P.L., Schmidt, K., Stark, E.L., Vogt, L.: Central factorial numbers; their main properties and some applications Numer. Funct. Anal. Optim. 10(5\&6), 419-488 (1989)

3. Cakic, N.P., Milovanovic, G.V.: On generalized Stirling numbers and polynomials. Math. Balk. 18, 241-248 (2004)

4. Cigler, J.: Fibonacci polynomials and central factorial numbers. Preprint

5. Comtet, L.: Advanced Combinatorics: The Art of Finite and Infinite Expansions. Reidel, Dordrecht (1974). Translated from the French by J.W. Nienhuys

6. Dere, R., Simsek, Y.: Hermite base Bernoulli type polynomials on the umbral algebra. Russ. J. Math. Phys. 22(1), 1-5 (2015)

7. Djordjevic, G.B., Milovanovic, G.V.: Special Classes of Polynomials. University of Nis, Faculty of Technology, Leskovac (2014)

8. El-Desouky, B.S., Mustafa, A.: New results and matrix representation for Daehee and Bernoulli numbers and polynomials. Appl. Math. Sci. 9, 3593-3610 (2015)

9. Khan, N., Usman, T., Choi, J.: A new class of generalized polynomials. Turk. J. Math. (2018, in press). https://doi.org/10.3906/mat-1709-44

10. Kim, D.S., Kim, T., Rim, S.-H., Lee, S.H.: Hermite polynomials and their applications associated with Bernoulli and Euler numbers. Discrete Dyn. Nat. Soc. 2012, Article ID 974632 (2012)

11. Kim, D.S., Kim, T.: Daehee numbers and polynomials. Appl. Math. Sci. (Ruse) 7, 5969-5976 (2013)

12. Kim, D.S., Kim, T., Seo, J.: A note on Changhee numbers and polynomials. Adv. Stud. Theor. Phys. 7, $993-1003$ (2013)

13. Kim, T:: $q$-Euler numbers and polynomials associated with $p$-adic $q$-integral and basic $q$-zeta function. Trend Math. Information Center Math. Sciences 9, 7-12 (2006)

14. Kim, T.: q-Volkenborn integration. Russ. J. Math. Phys. 19, 288-299 (2002)

15. Kim, T.: On the q-extension of Euler and Genocchi numbers. J. Math. Anal. Appl. 326(2), 1458-1465 (2007)

16. Kim, T., Rim, S.-H., Simsek, Y., Kim, D.: On the analogs of Bernoulli and Euler numbers, related identities and zeta and I-functions. J. Korean Math. Soc. 45(2), 435-453 (2008)

17. Kim, T., Choi, J., Kim, Y.-H.: Some identities on the $q$-Bernstein polynomials, $q$-Stirling numbers and $q$-Bernoulli numbers. arXiv:1006.2033v1

18. Luo, Q.M., Srivastava, H.M.: Some relationships between the Apostol-Bernoulli and Apostol-Euler polynomials. Comput. Math. Appl. 51(3-4), 631-642 (2006)

19. Milne-Thomson, L.M.: Two classes of generalized polynomials. Proc. Lond. Math. Soc. s2-35(1), 514-522 (1933)

20. Rainville, E.D.: Special Functions. Macmillan Co., New York (1960)

21. Riordan, J.: Introduction to Combinatorial Analysis. Princeton University Press, Princeton (1958)

22. Roman, S.: The Umbral Calculus. Dover, New York (2005)

23. Schikhof, W.H.: Ultrametric Calculus: An Introduction to $p$-Adic Analysis. Cambridge Studies in Advanced Mathematics, vol. 4. Cambridge University Press, Cambridge (1984)

24. Simsek, Y.: Generating functions for generalized Stirling type numbers, array type polynomials, Eulerian type polynomials and their alications. Fixed Point Theory Appl. 2013, 87 (2013)

25. Simsek, Y.: Identities associated with generalized Stirling type numbers and Eulerian type polynomials. Math. Comput. Appl. 18(3), 251-263 (2013). https://doi.org/10.3390/mca18030251

26. Simsek, Y.: Special numbers on analytic functions. Appl. Math. 5, 1091-1098 (2014

27. Simsek, Y.: Complete sum of products of $(h ; q)$-extension of Euler polynomials and numbers. J. Differ. Equ. Appl. 16 $1331-1348(2010)$

28. Simsek, Y.: Twisted $(h ; q)$-Bernoulli numbers and polynomials related to twisted $(h ; q)$-zeta function and $L$-function. J. Math. Anal. Appl. 324, 790-804 (2006)

29. Simsek, Y.: Formulas for Poisson-Charlier, Hermite, Milne-Thomson and other type polynomials by their generating functions and p-adic integral approach. Rev. R. Acad. Cienc. Exactas Fís. Nat., Ser. A Mat. (2018, in press). https://doi.org/10.1007/s13398-018-0528-6

30. Srivastava, H.M.: Some generalizations and basic (or $q^{-}$) extensions of the Bernoulli, Euler and Genocchi polynomials. Appl. Math. Inf. Sci. 5, 390-444 (2011) 
31. Srivastava, H.M., Choi, J.: Zeta and q-Zeta Functions and Associated Series and Integrals. Elsevier, Amsterdam (2012)

32. Srivastava, H.M., Liu, G.-D.: Some identities and congruences involving a certain family of numbers. Russ. J. Math. Phys. $16,536-542$ (2009)

33. Yuluklu, E.: A note on the Milne-Thomson type polynomials. In: AIP Conf. Proc. (2018, in press)

Submit your manuscript to a SpringerOpen ${ }^{\circ}$ journal and benefit from:

- Convenient online submission

- Rigorous peer review

- Open access: articles freely available online

- High visibility within the field

- Retaining the copyright to your article

Submit your next manuscript at $\boldsymbol{\nabla}$ springeropen.com 